\title{
STATE FEEDBACK FUZZY-MODEL-BASED CONTROL FOR MARKOVIAN JUMP NONLINEAR SYSTEMS
}

\author{
Natache S. D. Arrifano* \\ natacheadsel.eesc.usp.br
}

\author{
Vilma A. Oliveira* \\ vilmao@sel.eesc.usp.br
}

${ }^{*}$ Departamento de Engenharia Elétrica, Universidade de São Paulo, Av. Trabalhador São Carlense, 400

CEP 13566-590, São Carlos, SP, BRASIL

\begin{abstract}
This paper deals with the fuzzy-model-based control design for a class of Markovian jump nonlinear systems. A fuzzy system modeling is proposed to represent the dynamics of this class of systems. The structure of the fuzzy system is composed of two levels, a crisp level which describes the Markovian jumps and a fuzzy level which describes the system nonlinearities. A sufficient condition on the existence of a stochastically stabilizing controller using a Lyapunov function approach is presented. The fuzzy-model-based control design is formulated in terms of a set of linear matrix inequalities. Simulation results for a single-machine infinitebus power system which is modeled as a Markovian jump nonlinear system in the infinite-bus voltage are presented to illustrate the applicability of the technique.
\end{abstract}

KEYWORDS: Markovian jump nonlinear systems, Markovian jump fuzzy systems, fuzzy-model-based control, stochastic stabilizability.

\section{RESUMO}

Neste artigo, apresentam-se projetos de controladores fuzzy para uma classe de sistemas não-lineares com saltos Markovianos. Uma modelagem fuzzy é apresentada para representar esta classe de sistemas na vizinhança de pontos de operação escolhidos. A estrutura do sistema fuzzy é composta de dois níveis, um para descrição dos saltos Markovianos e ou-

Artigo submetido em 28/02/03

1a. Revisão em 15/01/04

Aceito sob recomendação do Ed. Assoc. Prof. Takashi Yoneyama tro para descrição das não-linearidades no estado do sistema. Uma condição suficiente para a estabilização estocástica do sistema fuzzy considerado é derivada usando uma função de Lyapunov acoplada. O projeto de controle fuzzy é então formulado a partir de um conjunto de desigualdades matriciais lineares. Resultados de simulações em um sistema de potência máquina-barramento infinito modelado como um sistema não-linear com saltos Markovianos na tensão do barramento infinito são apresentados para ilustrar a aplicabilidade da técnica.

PALAVRAS-CHAVE: Sistemas não-lineares com saltos Markovianos, sistemas fuzzy com saltos Markovianos, controle fuzzy, estabilização estocástica.

\section{INTRODUCTION}

The class of nonlinear systems considered in this paper is a class of hybrid systems, which has different operation modes governed by a Markov process. They are described by a state vector with two components where the first refers to the system modes and the second to the system state. The system modes are represented by a finite-mode Markov process and the system state in each mode by a system of nonlinear differential equations. This class of systems can be used to represent complex real systems, which may experience abrupt changes in their structure and parameters caused by phenomena such as component failures or repairs, changing of subsystem interconnections and abrupt environmental disturbances.

Because of the difficulties inherent in the analysis of non- 
linear dynamics, most attention has been given to the linear representation of Markovian jump systems. The Markovian jump linear systems (MJLS) were first introduced by Krasovskii and Lidskii (1961) and have been used to model manufacturing management systems, power systems, telecommunication and economic systems (Mariton, 1990). In this context, the linear quadratic control problem was addressed (Boukas and Liu, 2001; Costa et al., 1999; Mariton and Bertrand, 1985a; Mariton and Bertrand, 1985b; Sworder, 1969). Lately, considerable attention has been paid to the robust control, robust stochastic stability and stabilizability of jumping linear uncertain systems (Farias et al., 2000; Boukas et al., 1999; Boukas and Yang, 1999; Costa and Boukas, 1998). In general, the system uncertainties considered appear as norm-bounded uncertainties, which facilitates the extension of the deterministic robust and optimal control techniques to the Markovian jump linear systems. Despite this, a more realistic model should consider the nonlinearities of a real system. To the best of our knowledge, the control for Markovian jump nonlinear system (MJNLS) was only considered in Rishel (1975) wherein the optimal control problem is formulated in terms of dynamic programming.

Recently, there have been many successful applications of fuzzy control to nonlinear systems (Arrifano and Oliveira, 2002a; Arrifano and Oliveira, 2002b; Nascimento et al., 2002; Teixeira and Żak, 1999; Tanaka et al., 1998; Wang et al., 1996). In general, the fuzzy control design considers a nonlocal approach which is conceptually simple and straightforward, where linear feedback control techniques can be used (Wang et al., 1996). To accomplish this, the nonlinear system is represented by a Takagi-Sugeno (TS) fuzzy system (Takagi and Sugeno, 1985), which is described by fuzzy IF-THEN rules representing local input-output relations of the nonlinear system. The basic idea of this approach is to decompose the input space into many subspaces, approximating the nonlinear system by a fuzzy blending of local linear systems associated to each subspace. In fact, it is proved that the TS fuzzy systems are universal approximators (Tanaka and Wang, 2001).

The fuzzy-model based control design uses the so-called parallel distributed compensation (PDC) scheme and Lyapunov stability. The idea of the PDC scheme is that a linear control is designed for each local linear system. The overall controller is again a fuzzy blending of all local linear controllers, which is nonlinear in general. This approach requires a common positive definite matrix that is a solution of all the Lyapunov inequalities built from the local linear systems of the global feedback TS fuzzy system, which are usually formulated in terms of linear matrix inequalities (LMI's) in both the feedback control gain and Lyapunov matrix. However, for a large number of local linear approximations this approach may not provide feasible results because it is not possible to find a common positive definite Lyapunov matrix as a solution of several Lyapunov inequalities. In order to relax the conservativeness of the stability and stabilization problems, piecewise Lyapunov function approaches have received increasing attention (Cao et al., 1997; Cao et al., 1996). With the same purposes, a fuzzy Lyapunov function defined by a fuzzy blending quadratic Lyapunov functions is considered in (Tanaka et al., 2003). The fuzzy Lyapunov function, unlike the piecewise Lyapunov function is smooth.

In this paper, we consider the use of two different fuzzymodel-based control designs for stochastic stabilization of a class of MJNLS. We propose a fuzzy system modeling with two levels of structure, a crisp level which describes the jumps of the Markov process and a fuzzy level which describes the system nonlinearities. Using the state feedback fuzzy system and a coupled Lyapunov function, we formulate a control design in terms of LMI's and the stochastic stabilizability concept. The remainder of this paper is organized as follows. Section 2 introduces the fuzzy system modeling. Section 3 presents the fuzzy-model-based control. Section 4 deals with the stabilizing fuzzy control design. Simulation results are presented in Section 5 to illustrate the applicability of the proposed approach. Concluding remarks are presented in Section 6.

\section{FUZZY SYSTEM MODELING}

Consider a class of Markovian jump nonlinear dynamic systems depicted by

$$
\dot{x}=f(x, u, r) ; x_{0}=x(0) ; r_{0}=r(0)
$$

where $x \in \mathbb{R}^{n}$ is the system state vector, $u \in \mathbb{R}^{m}$ is the control input vector, $\{r\}$ is a continuous-time Markovian process taking values in a finite space state denoted by $\mathbb{S}=\{1,2, \ldots, N\}, f(\cdot, \cdot, \cdot)$ is a smooth nonlinear function with respect to the first and the second arguments with $f(0,0,)=0,. x_{0}$ and $r_{0}$ are the initial values of the state and the mode at time $t=0$, respectively. The evolution of the stochastic process $\{r, t \geq 0\}$ that determines the mode of the system at each time $t$ is assumed to be described by the following transition probability

$\operatorname{Pr}\{r(t+\Delta)=j \mid r(t)=i\}:=\left\{\begin{array}{l}\pi_{i j} \Delta+o(\Delta), i \neq j \\ 1-\pi_{i} \Delta+o(\Delta), i=j\end{array}\right.$

where $\Delta>0, \lim _{\Delta \rightarrow 0} O(\Delta) \Delta^{-1}=0, \pi_{i j} \geq 0$ is the probability rate between modes $i$ and $j$, for $i \neq j ; i, j \in \mathbb{S}$ and $\forall i \in \mathbb{S}, \pi_{i}:=-\pi_{i i}=\sum_{j=1, j \neq i}^{N} \pi_{i j}$. A matrix $\Pi:=\left[\pi_{i j}\right]$ is called transition rate matrix. We assume that the Markov process $\{r\}$ has stationary distribution $\mu=\left(\mu_{1}, \mu_{2}, \cdots, \mu_{N}\right)$ with $\mu_{i}=\operatorname{Pr}(r=i)$

In order to model jumps in a fuzzy system modeling, we propose a Markovian jump fuzzy system (MJFS) following the 
idea of a switching fuzzy system (SFS) proposed by Tanaka et al. (2000). The SFS have a region rule level which is crisp and a local rule level which is fuzzy. Likewise, the MJFS is structured in upper and lower levels for the modes assumed by the Markov process $\{r\}$ and for the fuzzy rule in each mode which describes the nonlinearities in the state vector $x$, respectively. Thus, the $i$ th mode assumed by the MJNLS is represented as follows

\section{Mode $i$ :}

If $z$ is $M_{i}$

Then

Rule $j$ :

$$
\begin{aligned}
& \text { If } x_{1} \text { is } N_{i j 1} \text { and } \ldots \text { and } x_{n} \text { is } N_{i j n} \\
& \text { Then } \dot{x}=A_{i j} x+B_{i j} u \\
& i \in \mathbb{S} ; j=1,2, \ldots, R
\end{aligned}
$$

where $z \in \mathbb{R}^{1}$ is a mode indicator variable, $x$ and $u$ are as defined before, $A_{i j}$ and $B_{i j}$ are matrices of appropriate dimensions, which describe local linear representations of the nonlinear system in the vicinity of chosen operation points, $M_{i}$ and $N_{i j k}$ are crisp and fuzzy sets, respectively, and $R$ is the number of inference rules in each mode. In the framework of fuzzy systems, the IF-part of the MJFS is referred to as the premise part and the THEN-part is referred to as the consequent part, variables $x$ and $z$ in the IF-part are known as premise variables. Usually, the premise variables may be functions of state variables, external disturbances, and/or time (Li et al., 2000).

Thus, the MJFS is inferred by a fuzzy blending of the local linear representations $\left(A_{i j}, B_{i j}\right), i \in \mathbb{S}, j=1,2, \ldots, R$, which are selected according to the mode assumed by the Markov process $\{r\}$. Thus, given the triple $(x, u, r)$, the overall fuzzy system is inferred as follows

$$
\begin{aligned}
\dot{x} & =\hat{f}(x, u, r) \\
& =\sum_{i=1}^{N} \sum_{j=1}^{R} m_{i}(z) n_{i j}(x)\left(A_{i j} x+B_{i j} u\right)
\end{aligned}
$$

where $m_{i}(z)$ is the mode indicator which yields $m_{i}(z)=1$ when $r=i$, i.e., $z \in M_{i}$ and $m_{i}(z)=0$ otherwise, and $n_{i j}(x)$ normalized membership functions given by

$$
n_{i j}(x)=\frac{\prod_{k=1}^{n} N_{i j k}\left(x_{k}\right)}{\sum_{l=1}^{R} \prod_{k=1}^{n} N_{i l k}\left(x_{k}\right)}
$$

with $N_{i j k}(x) \in[0,1]$ the grade of membership of $x_{k}, k=$ $1,2, \ldots, n$ in the fuzzy set $N_{i j k}$. In addition, considering the fact that in (5) $N_{i j k}\left(x_{k}\right) \geq 0, j=1,2, \ldots, R$, we have $n_{i j}(x) \geq 0$ and $\sum_{j=1}^{R} n_{i j}(x)=1$.
The universe of discourse $\mathbb{X}: \mathbb{R}^{n} \times \mathbb{S} \rightarrow \mathbb{R}^{n}$ for the MJFS is given by

$$
\begin{gathered}
\mathbb{X}=\cup_{i=1}^{N} \text { Mode } i=\text { Mode } 1 \cup \text { Mode } 2 \cup \ldots \cup \text { Mode } N \\
\text { Mode } i \cap \text { Mode } \ell=\phi, i \neq \ell, i, \ell \in \mathbb{S} .
\end{gathered}
$$

Remark 1 The local linear representations of the MJFS can be constructed via the linearization formula proposed by Teixeira and Żak (1999) which yields a good linear approximation of the nonlinear system in the vicinity of a specified operation point even if it is not an equilibrium point.

\section{FUZZY-MODEL-BASED CONTROL}

The fuzzy-model-based control is in general developed using the PDC scheme. Following this trend, the fuzzy controller proposed here shares the same structure of the MJFS (3) in its premise part, i.e.,

\section{Mode $i$ :}

$$
\text { If } z \text { is } M_{i}
$$

Then

Rule $j$ :

$$
\begin{aligned}
& \text { If } x_{1} \text { is } N_{i j 1} \text { and } \ldots \text { and } x_{n} \text { is } N_{i j n} \\
& \text { Then } u=-F_{i j} x \\
& i \in \mathbb{S} ; j=1,2, \ldots, R
\end{aligned}
$$

where $z, x, M_{i}, N_{i j}$ and $R$ are as defined before and $F_{i j} \in$ $\mathbb{R}^{m \times n}$ are the local feedback gains to be designed. Following the same lines as in the derivation of the MJFS, we obtain the overall fuzzy controller as

$$
u=-\sum_{i=1}^{N} \sum_{j=1}^{R} m_{i}(z) n_{i j}(x) F_{i j} x .
$$

In order to obtain the state feedback MJFS as following, we substitute (7) in (4), it results

$$
\begin{aligned}
\dot{x}= & \sum_{i=1}^{N} \sum_{j=1}^{R} m_{i}(z) n_{i j}(x)\left[A_{i j}\right. \\
& \left.-\left(\sum_{k=1}^{N} \sum_{l=1}^{R} m_{k}(z) n_{k l}(x) B_{i j} K_{k l}\right)\right] x .
\end{aligned}
$$

Using the fact that $m_{i}(z) m_{k}(z)=0, i \neq k, i, k \in \mathbb{S}$, we can write (8) as

$$
\dot{x}=\sum_{i=1}^{N} m_{i}(z)\left[\sum_{j=1}^{R} \sum_{k=1}^{R} n_{i j}(x) n_{i k}(x)\left(A_{i j}-B_{i j} K_{i k}\right)\right] x .
$$


Now, using the fact that

$$
\sum_{j=1}^{R} \sum_{k=1}^{R} n_{i j}(x) n_{i k}(x)=\sum_{j=1}^{R} n_{i j}^{2}(x)+2 \sum_{j<k}^{R} n_{i j}(x) n_{i k}(x)
$$

and $\sum_{j=1}^{R} n_{i j}(x)=1$, system (9) can be rewritten as

$$
\dot{x}=\sum_{i=1}^{N} m_{i}(z)\left[\sum_{j=1}^{R} n_{i j}^{2}(x) G_{i j}+2 \sum_{j<k}^{R} n_{i j}(x) n_{i k}(x) H_{i j k}\right] x
$$

with $G_{i j}:=A_{i j}-B_{i j} F_{i j}$ and $H_{i j k}:=\frac{1}{2}\left(A_{i j}-B_{i j} F_{i k}+\right.$ $\left.A_{i k}-B_{i k} F_{i j}\right), i \in \mathbb{S}, j, k=1,2, \ldots, R$. In (10), notation $\sum_{j<k}^{R}$ means, for instance for $R=3, \sum_{j<k}^{3} a_{j k} \Leftrightarrow a_{12}+$ $a_{13}+a_{23}$.

Remark 2 The use of (10) instead of (9) is valuable to reduce the number of LMI's conditions in the formulation of the fuzzy control design.

\section{STABILIZING FUZZY CONTROL DESIGN}

In this section, we present a sufficient condition for the stochastic stabilization of the MJFS using a coupled Lyapunov function. In order to obtain a systematic fuzzy control design, we formulate the stabilizing control problem in the context of the convex analysis using LMI's. In the following, $E[\cdot]$ denotes the expectancy operator and $\lambda_{\min }[\cdot]$ and $\lambda_{\max }[\cdot]$ denote the minimum and the maximum eigenvalues, respectively.

Definition 1 The MJFS (4) with infinitesimal generator $\mathcal{A}$ is exponentially stable in mean square (ESMS) if there exists a coupled Lyapunov function of the type

$$
V(x, i)=x^{T} P_{i} x
$$

$\forall i \in \mathbb{S}$ with $P_{i}:=P_{r=i}$ a symmetric positive definite constant matrix of appropriate dimensions such that

1. $V(0, r=i)=0$;

2. $V(\cdot, \cdot)$ is continuous and has bounded first derivatives with respect to the first argument;

3. $c_{1}\|x\|^{2} \leq V(x, i) \leq c_{2}\|x\|^{2}$;

4. $\mathcal{A} V(x, i) \leq-c_{3}\|x\|^{2}$;

for $c_{1}, c_{2}$ and $c_{3}$ positive real numbers (Mariton, 1990).
Definition 2 The MJFS (4) is said to be stochastically stable if, for all the initial conditions $x_{0}$ and $r_{0}$ there exists a state feedback fuzzy control law (7) satisfying

$$
\begin{array}{r}
\lim _{T \rightarrow \infty} E\left[\int_{0}^{T} x\left(t, x_{0}, r_{0}, u\right)^{T} x\left(t, x_{0}, r_{0}, u\right) d t \mid x_{0}, r_{0}\right] \\
\leq x_{0}^{T} M x_{0}
\end{array}
$$

for some symmetric positive definite matrix $M$ of appropriate dimensions (Ji and Chizeck, 1990).

Proposition 1 The MJFS (4) is stochastically stabilizable with state feedback fuzzy control law (7) if there exist a set of positive definite matrices $X_{i}$ and a set of matrices $Y_{i j}$ of appropriate dimensions satisfying the following LMI's $\forall i \in \mathbb{S}$

$$
\begin{array}{r}
{\left[\begin{array}{ll}
T_{i j} & Z_{i} \\
Z_{i}^{T} & -W_{i}
\end{array}\right]<0} \\
\quad j=1,2, \ldots, R
\end{array}
$$

and

$$
\begin{array}{r}
{\left[\begin{array}{cc}
U_{i j k} & Z_{i} \\
Z_{i}^{T} & -W_{i}
\end{array}\right]<0 ;} \\
j<k ; j, k=1,2, \ldots, R
\end{array}
$$

where

$T_{i j}:=X_{i} A_{i j}^{T}+A_{i j} X_{i}-Y_{i j}^{T} B_{i j}^{T}-B_{i j} Y_{i j}-\frac{1}{2} \pi_{i} X_{i}$

$U_{i j k}:=X_{i} A_{i j}^{T}+A_{i j} X_{i}-Y_{i k}^{T} B_{i j}^{T}-B_{i j} Y_{i k}$

$\begin{aligned} U_{i j k}:= & +X_{i} A_{i k}^{T}+A_{i k} X_{i}-Y_{i j}^{T} B_{i k}^{T}-B_{i k} Y_{i j}-\frac{1}{2} \pi_{i} X_{i}\end{aligned}$

$Z_{i}:=\left[\begin{array}{llllll}\pi_{i 1}^{1 / 2} X_{i} & \ldots & \pi_{i i-1}^{1 / 2} X_{i} & \pi_{i i+1}^{1 / 2} X_{i} & \ldots & \pi_{i N}^{1 / 2} X_{i}\end{array}\right]$

$W_{i}:=\operatorname{diag}\left\{\begin{array}{llllll}X_{1} & \ldots & X_{i-1} & X_{i+1} & \ldots & X_{N}\end{array}\right\}$

$Y_{i j}:=F_{i j} X_{i}$

$X_{i}:=P_{i}^{-1}$.

Proof: Let mode at time $t$ be $i$, i.e., $r=i, i \in \mathbb{S}$. In what follows, for simplicity of notation $x$ denotes the solution $x\left(t, x_{0}, r_{0}, u\right)$ of the MJFS (4) under the initial conditions $x_{0}$ and $r_{0}$ with fuzzy control law (7).

Take the coupled Lyapunov function as in (10). The weak infinitesimal operator of (10) is given by (Ji and Chizeck, 1990)

$$
\begin{aligned}
\mathcal{A} V(x, i):= & \lim _{\delta \rightarrow 0} \frac{1}{\delta}\{E[V(x(t+\delta), r(t+\delta)) \mid x, r=i] \\
& -V(x, r=i)\} .
\end{aligned}
$$

The weak infinitesimal operator $\mathcal{A}$ of a function of the joint stochastic process $\{x, r\}$ is the natural stochastic analog of 
the deterministic derivative. Using Mariton (1990), from (13) it is possible to obtain

$$
\begin{aligned}
\mathcal{A} V(x, i) & =\dot{x}^{T} \frac{\partial}{\partial x} V(x, i)+\sum_{\ell=1}^{N} \pi_{i \ell} V(x, \ell) \\
& =\dot{x}^{T} P_{i} x+x^{T} P_{i} \dot{x}+x^{T}\left(\sum_{\ell=1}^{N} \pi_{i \ell} P_{i}\right) x .
\end{aligned}
$$

Substituting (10) in (14) and using the fact that $m_{i}(z)=1$ when $z \in M_{i}$, we obtain

$$
\begin{aligned}
\mathcal{A} V(x, i)= & x^{T}\left[\sum_{j=1}^{R} n_{i j}^{2}(x)\left(G_{i j}^{T} P_{i}+P_{i} G_{i j}\right)\right. \\
& \left.+2 \sum_{j<k}^{R} n_{i j}(x) n_{i k}(x)\left(H_{i j k}^{T} P_{i}+P_{i} H_{i j k}\right)\right] x \\
& +x^{T}\left(\sum_{\ell=1}^{N} \pi_{i \ell} P_{i}\right) x
\end{aligned}
$$

for $G_{i j}$ and $H_{i j k}$ as defined before. Now, using the Schur complements (Boyd et al., 1994) and substituting $T_{i j}, U_{i j k}$, $Z_{i}, W_{i}, Y_{i j}$ and $X_{i}$ as defined before, LMI's in (12) can be reduced to

$$
\begin{array}{r}
G_{i j}^{T} P_{i}+P_{i} G_{i j}+\sum_{\substack{\ell=1 \\
N}}^{N} \pi_{i \ell} P_{\ell}<0 \\
\quad j=1,2, \ldots, R
\end{array}
$$

and

$$
\begin{array}{r}
H_{i j k}^{T} P_{i}+P_{i} H_{i j k}+\sum_{\ell=1}^{N} \pi_{i \ell} P_{\ell}<0 ; \\
j<k ; j, k=1,2, \ldots, R .
\end{array}
$$

Multiplying (16a) by $n_{i j}^{2}(x)$ and (16b) by $2 n_{i j}(x) n_{i k}(x)$, we have

$$
\begin{aligned}
& \sum_{j=1}^{R} n_{i j}^{2}(x)\left[G_{i j}^{T} P_{i}+P_{i} G_{i j}\right] \\
& +\sum_{j=1}^{R} n_{i j}^{2}(x) \sum_{\ell=1}^{N} \pi_{i \ell} P_{\ell}<0
\end{aligned}
$$

and

$$
\begin{array}{r}
2 \sum_{j<k}^{R} n_{i j}(x) n_{i k}(x)\left[H_{i j k}^{T} P_{i}+P_{i} H_{i j k}\right] \\
\quad+2 \sum_{j<k}^{R} n_{i j}(x) n_{i k}(x) \sum_{\ell=1}^{N} \pi_{i \ell} P_{\ell}<0 .
\end{array}
$$

Now, adding (17a) to (17b) and again using the fact that

$$
\sum_{j=1}^{R} \sum_{k=1}^{R} n_{i j}(x) n_{i k}(x)=\sum_{j=1}^{R} n_{i j}^{2}(x)+2 \sum_{j<k}^{R} n_{i j}(x) n_{i k}(x)
$$

and $\sum_{j=1}^{R} n_{i j}(x)=1$, we obtain $\mathcal{A} V(x, i)<0$ for $x \neq 0$.

Now, defining

$$
\begin{aligned}
& \mathcal{L}\left(\bar{G}_{i j}, \bar{H}_{i j k}, P_{i}\right):=\bar{G}_{i j}^{T} P_{i}+P_{i} \bar{G}_{i j} \\
& +2\left(\bar{H}_{i j k}^{T} P_{i}+P_{i} \bar{H}_{i j k}\right)+\sum_{\ell=1}^{N} \pi_{i \ell} P_{\ell}
\end{aligned}
$$

with $\bar{G}_{i j}=\sum_{j=1}^{R} n_{i j}^{2}(x) G_{i j}$ and $\bar{H}_{i j k}=\sum_{j<k}^{R}$ $n_{i j}(x) n_{i k}(x) H_{i j k}$ and substituting (18) in (15), we obtain

$$
\mathcal{A} V(x, i)=x^{T} \mathcal{L}\left(\bar{G}_{i j}, \bar{H}_{i j k}, P_{i}\right) x .
$$

Therefore, we have for all $x \neq 0$ and $i \in \mathbb{S}$

$$
\begin{aligned}
\frac{\mathcal{A} V(x, i)}{V(x, i)} & =\frac{x^{T} \mathcal{L}\left(\bar{G}_{i j}, \bar{H}_{i j k}, P_{i}\right) x}{x^{T} P_{i} x} \\
& \leq-\rho
\end{aligned}
$$

where $\rho$ is a positive real number given by

$$
\rho=\min _{i \in \mathbb{S}} \frac{\lambda_{\min }\left[-\mathcal{L}\left(\bar{G}_{i j}, \bar{H}_{i j k}, P_{i}\right)\right]}{\lambda_{\max }\left[P_{i}\right]} .
$$

By the Dynkin's formula (Kushner, 1967), we have

$E[V(x(t), r(t))]-V\left(x_{0}, r_{0}\right)=E\left[\int_{0}^{t} \mathcal{A} V(x(s), r(s)) d s\right]$.

Then, substituting (20) in (22), we obtain

$$
\begin{aligned}
& E[V(x(t), r(t))]-V\left(x_{0}, r_{0}\right) \\
& \leq E\left[\int_{0}^{t}-\rho V(x(s), r(s)) d s\right] \\
& =-\rho \int_{0}^{t} E[V(x(s), r(s)) d s] .
\end{aligned}
$$

Using the Gronwall-Bellman Lemma (Khalil, 1996) in (23), we have

$$
E[V(x(t), r(t))] \leq V\left(x_{0}, r_{0}\right) \exp (-\rho t) .
$$

Integrating both sides of (24) and taking the limit as $T \rightarrow \infty$, 
it results

$$
\begin{aligned}
\lim _{T \rightarrow \infty} E\left[\int_{0}^{T} x^{T} P_{r} x d t \mid x_{0}, r_{0}\right] & \leq \frac{1}{\rho} x_{0}^{T} P_{r} x_{0} \\
& \leq \frac{1}{\rho} \lambda_{\max }\left[P_{r}\right] x_{0}^{T} x_{0} .
\end{aligned}
$$

Considering the fact that in (25) $P_{r}$ is a symmetric positive definite matrix for all $r \in \mathbb{S}$, the result follows by Definitions 1 and 2 .

\subsection{Performance indices in the fuzzy con- trol design}

Like stability, performance indices, such as decay rate and control input, play a key role in the stabilizing fuzzy control design. The speed response of a controlled system is related to decay rate, that is, the largest Lyapunov exponent. In addition, there are some applications in real systems, where the control input has to be limited to guarantee the system operation conditions. In what follows, we formulate the stabilizing fuzzy control design using the decay rate $\alpha_{i}:=\alpha_{r=i}$ and control input $\gamma_{i}: \gamma_{r=i}, i \in \mathbb{S}$ in the context of LMI's.

Proposition 2 Assume that the decay rate $\alpha_{i}>0, i \in \mathbb{S}$ is known. The condition

$$
\mathcal{A} V(x, i) \leq-2 \alpha_{i} V(x, i)
$$

is enforced to all trajectories of the MJFS (4) with state feedback fuzzy control law (7), if there exist a set of positive definite matrices $X_{i}$ and a set of matrices $Y_{i j}$ of appropriate dimensions satisfying the following LMI's $\forall i \in \mathbb{S}$

$$
\begin{array}{r}
{\left[\begin{array}{ll}
T_{i j} & Z_{i} \\
Z_{i}^{T} & -W_{i}
\end{array}\right]<-2 \alpha_{i}\left[\begin{array}{ll}
X_{i} & 0 \\
0 & 0
\end{array}\right]} \\
j=1,2, \ldots, R
\end{array}
$$

and

$$
\begin{array}{r}
{\left[\begin{array}{ll}
U_{i j k} & Z_{i} \\
Z_{i}^{T} & -W_{i}
\end{array}\right]<-2 \alpha_{i}\left[\begin{array}{ll}
X_{i} & 0 \\
0 & 0
\end{array}\right]} \\
j<k ; j, k=1,2, \ldots, R
\end{array}
$$

where $T_{i j}, U_{i j k}, Z_{i}, W_{i}, X_{i}$ and $Y_{i j}$ are as defined before.

Proof: The proof follows the same lines of the proof of Proposition 1.

Proposition 3 Assume that the initial condition $x_{0}$ is known. The constraint

$$
E\left[u^{T} u \mid x, r=i\right] \leq \gamma_{i}^{2}
$$

is enforced to all trajectories of the MJFS (4) with state feedback fuzzy control law (7), if the following LMI's hold $\forall i \in \mathbb{S}$

$$
\left[\begin{array}{ll}
1 & x_{0}^{T} \\
x_{0} & X_{i}
\end{array}\right] \geq 0
$$

and

$$
\begin{aligned}
& {\left[\begin{array}{cc}
X_{i} & Y_{i j}^{T} \\
Y_{i j} & \gamma_{i} I
\end{array}\right] \geq 0} \\
& \quad j=1,2, \ldots, R
\end{aligned}
$$

where $X_{i}$ and $Y_{i j}$ are as defined before.

Proof: Assume that $V(x, i)$ in (10) is a Lyapunov function for all trajectories of the MJFS (4) with state feedback fuzzy control law (7). Substituting (7) in (28) and using the fact that $m_{i}(z) m_{\ell}(z)=0, i \neq \ell, i, \ell \in \mathbb{S}$, we have

$E\left[\sum_{i=1}^{R} m_{i}^{2}(z)\left(\sum_{j=1}^{R} \sum_{k=1}^{R} n_{i j}(x) n_{i k}(x) x^{T} F_{i j}^{T} F_{i j} x\right)\right] \leq \gamma_{i}^{2}$.

Let the mode at time $t$ be $i$, i.e., $r=i, i \in \mathbb{S}$. Thus, (30) can be written as

$$
\sum_{j=1}^{R} \sum_{k=1}^{R} n_{i j}(x) n_{i k}(x) x^{T}\left(\frac{1}{\gamma_{i}^{2}} F_{i j}^{T} F_{i j}\right) x \leq 1 .
$$

Now, we use (29a) in order to obtain (31). Using the Schur complements in (29a) and (29b), it results for all $i \in \mathbb{S}$

$$
x_{0}^{T} P_{i} x_{0} \leq 1
$$

and

$$
\begin{array}{r}
\frac{1}{\gamma^{2}} F_{i j}^{T} F_{i j}-P_{i} \leq 0 \\
j=1,2, \ldots, R .
\end{array}
$$

Multiplying (32b) by $n_{i j}(x)$ and using the fact that $\sum_{j=1}^{R} n_{i j}(x)=1$, we obtain

$$
\sum_{j=1}^{R} n_{i j}(x) x^{T}\left(\frac{1}{\gamma_{i}^{2}} F_{i j}^{T} F_{i j}-P_{i}\right) x \leq 0 .
$$

It can be shown that (Tanaka and Wang, 2001)

$$
\begin{array}{r}
\sum_{j=1}^{R} \sum_{k=1}^{R} n_{i j}(x) n_{i k}(x) x^{T}\left(\frac{1}{\gamma^{2}} F_{i j}^{T} F_{i j}-P_{i}\right) x \\
\leq \sum_{j=1}^{R} n_{i j}(x) x^{T}\left(\frac{1}{\gamma_{i}^{2}} F_{i j}^{T} F_{i j}-P_{i}\right) x .
\end{array}
$$


Thus, using (33) in (34), we have

$$
\sum_{j=1}^{R} \sum_{k=1}^{R} n_{i j}(x) n_{i k}(x) x^{T}\left(\frac{1}{\gamma^{2}} F_{i j}^{T} F_{i j}-P_{i}\right) x \leq 0
$$

which is the same as

$$
\sum_{j=1}^{R} \sum_{k=1}^{R} n_{i j}(x) n_{i k}(x) x^{T}\left(\frac{1}{\gamma^{2}} F_{i j}^{T} F_{i j}\right) x \leq x^{T} P_{i} x .
$$

Finally, as $V(x, i) \leq x_{0}^{T} P_{i} x_{0}$ by (32a), from (36), we obtain (31) and the result follows.

A stabilizing control design with the decay rate and the control input constraints can be defined as follows: Find a set of positive definite matrices $X_{i}$ and a set of matrices $Y_{i j}$ of appropriate dimensions satisfying (27) and (29) $\forall i \in \mathbb{S}$.

Remark 3 In the approach given, the fuzzy-model-based control design is based on the matrices $\left(A_{i j}, B_{i j}, \Pi\right), i \in \mathbb{S}$, $j=1,2, \ldots, R$. Thus, the control design based on LMI's conditions is strongly related to the number of inference rules and to the modes assumed by the Markov process. The properties of the normalized membership functions can be explored in order to reduce the number of intersections among the fuzzy sets and thus producing more relaxed LMI conditions. Examples of fuzzy-model-based control design using relaxed LMI conditions are given in Teixeira et al. (2003), Teixeira et al. (2000), and Tanaka et al. (1998).

Remark 4 In order to consider the stochastic stabilization of the MJNLS in case the equilibrium point is not the origin, that is, $(x, u) \neq 0$, one should perform a change of coordinates to make the origin the new equilibrium, before designing the fuzzy control (7) using Propositions 1, 2 and 3.

\section{SIMULATION RESULTS}

In this section, an illustrative example of the application of the developed approach is given. We consider the same example as in Guo et al. (2001), a single-machine-infinite-bus (SMIB) power system shown in Figure 1. The dynamic operation of the SMIB power system was modeled as being a Markovian jump nonlinear system described by

$$
\begin{aligned}
\dot{x}_{1}= & x_{2} \\
\dot{x}_{2}= & -\frac{D}{2 H} x_{2}+\frac{\omega_{0}}{2 H}\left(P_{m}-x_{3}\right) \\
\dot{x}_{3}= & \frac{x_{d s}}{x_{d s}^{\prime} T_{d o}^{\prime}}\left[T_{d o}^{\prime}\left(x_{d}-x_{d}^{\prime}\right)\left(\frac{z \sin \left(x_{1}\right)}{x_{d s}}\right)^{2} x_{2}+P_{m}-x_{3}\right] \\
& +\frac{\cos \left(x_{1}\right)}{\sin \left(x_{1}\right)} x_{2} x_{3}+\frac{x_{d s}}{x_{d s}^{\prime} T_{d o}^{\prime}}\left(\frac{z \sin \left(x_{1}\right)}{x_{d s}}\right) k_{c} u
\end{aligned}
$$

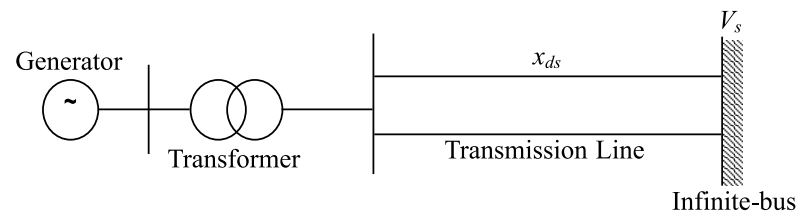

Figure 1: Single-machine-infinite-bus power system.

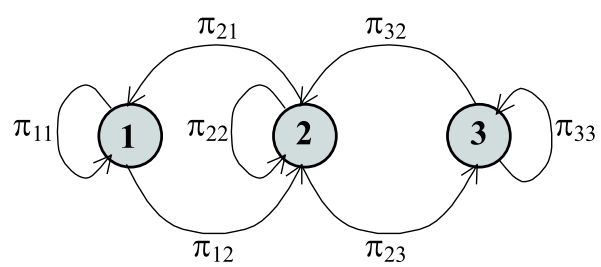

Figure 2: Transition modes of the SMIB power system.

where $\{x, z\}$ is a joint Markov process with stationary distribution $\mu=(0.3,0.5,0.2), x_{1}$ the power angle of the generator [rad], $x_{2}$ the relative speed of the generator [ $\left.\mathrm{rad} / \mathrm{s}\right], x_{3}$ the active power delivered to bus [p.u.], $u$ the input voltage of the SCR amplifier of the generator [p.u.], $z:=V_{s}$ the infinite bus voltage [p.u.], $D$ the damping constant [p.u.], $H$ the inertia constant $[\mathrm{s}], \omega_{0}$ the synchronous machine speed [rad/s], $P_{m}$ the mechanical input power [p.u.], $T_{d o}^{\prime}$ the direct axis transient short-circuit time constant [s], $x_{d}, x_{d}^{\prime}, x_{d s}$ and $x_{d s}^{\prime}$ the system reactances [p.u.]. In the simulations, we adopt the following numerical values of the physical parameters: $D=5, H=4, \omega_{0}=314.159, T_{d o}^{\prime}=6.9, K_{c}=1$, $x_{d}=1.8623, x_{d}^{\prime}=0.257, x_{d s}=2.4753$ and $x_{d s}^{\prime}=0.8693$.

System (37) presents the following equilibrium point $x_{e}=$ $\left[\begin{array}{lll}2 \pi / 5 & 0 & 0.9\end{array}\right]^{T}$ and $u_{e}=0$. As mentioned in Remark 4, it is necessary to perform a change of coordinates to bring the equilibrium of the system (37) to the origin. For this purpose, we adopt $\xi=x-x_{e}$ and $v=u-u_{e}$. Using these new coordinates, we may write (37) as

$$
\begin{aligned}
\dot{\xi}_{1}= & \xi_{2} \\
\dot{\xi}_{2}= & -\frac{D}{2 H} \xi_{2}+\frac{\omega_{0}}{2 H}\left(\xi_{3}+0.9\right) \\
\dot{\xi}_{3}= & \frac{x_{d s}}{x_{d s}^{\prime} T_{d o}^{\prime}}\left[T_{d o}^{\prime}\left(x_{d}-x_{d}^{\prime}\right)\left(\frac{z \sin \left(\xi_{1}+2 \pi / 5\right)}{x_{d s}}\right)^{2} \xi_{2}\right] \\
& -\frac{x_{d s}}{x_{d s}^{\prime} T_{d o}^{\prime}}\left(\xi_{3}+0.9\right)+\frac{\cos \left(\xi_{1}+2 \pi / 5\right)}{\sin \left(\xi_{1}+2 \pi / 5\right)} \xi_{2}\left(\xi_{3}+0.9\right) \\
& +\frac{x_{d s}}{x_{d s}^{\prime} T_{d o}^{\prime}}\left(\frac{z \sin \left(\xi_{1}+2 \pi / 5\right)}{x_{d s}}\right) k_{c} v .
\end{aligned}
$$

The infinite-bus voltage $z$ is modeled as a Markov chain with three different modes $(N=3)$ corresponding to the influence of an external disturbance in the equivalent load of the infinite-bus as following, mode 1: 1.2144 p.u. (low load), 
mode 2: 1.1040 p.u. (normal load) and mode 3: 0.9936 p.u. (heavy load). The transitions among the modes are illustrated in Figure 2. In accordance with the stationary distribution $\mu$ for the Markov process $r$, we adopt the following transition probability rate matrix for $z$

$$
\Pi=\left[\begin{array}{rrr}
0.1 & -0.1 & 0 \\
-0.06 & 0.1 & -0.04 \\
0 & -0.1 & 0.1
\end{array}\right]
$$

In order to obtain the local linear representations of system (38) in each mode, we adopt $R=2$ and consider deviations of $\pm \pi / 5$ in $x_{1}$, which gives the following linearization points $\bar{x}$ : mode $1: \bar{x}_{R=1}=\left[\begin{array}{lll}\pi / 5 & 0 & 1.089\end{array}\right]$ and $\bar{x}_{R=2}=\left[\begin{array}{lll}3 \pi / 5 & 0 & 1.089\end{array}\right]$, mode $2: \bar{x}_{R=1}=\left[\begin{array}{lll}\pi / 5 & 0 & 0.9\end{array}\right]$ and $\bar{x}_{R=2}=\left[\begin{array}{lll}3 \pi / 5 & 0 & 0.9\end{array}\right]$ and mode $3: \bar{x}_{R=1}=\left[\begin{array}{lll}\pi / 5 & 0 & 0.729\end{array}\right]$ and $\bar{x}_{R=2}=\left[\begin{array}{lll}3 \pi / 5 & 0 & 0.729\end{array}\right]$. Thus, using the procedure given in the Appendix, the following matrices $\left(A_{i j}, B_{i j}\right), i=1,2,3, j=1,2$ for the SMIB system are obtained

$$
\begin{aligned}
& A_{11}=\left[\begin{array}{rrr}
0 & 1.0000 & 0 \\
0 & -0.6250 & -39.2699 \\
0 & 1.8792 & -0.4127
\end{array}\right] ; B_{11}=\left[\begin{array}{r}
0 \\
0 \\
0.1190
\end{array}\right] ; \\
& A_{12}=\left[\begin{array}{rrr}
0 & 1.0000 & 0 \\
0 & -0.6250 & -39.2699 \\
0 & 0.6418 & -0.4127
\end{array}\right] ; B_{12}=\left[\begin{array}{r}
0 \\
0 \\
0.1926
\end{array}\right] ; \\
& A_{21}=\left[\begin{array}{rrr}
0 & 1.0000 & 0 \\
0 & -0.6250 & -39.2699 \\
0 & 1.5530 & -0.4127
\end{array}\right] ; B_{21}=\left[\begin{array}{r}
0 \\
0 \\
0.1082
\end{array}\right] ; \\
& A_{22}=\left[\begin{array}{rrr}
0 & 1.0000 & 0 \\
0 & -0.6250 & -39.2699 \\
0 & 0.5304 & -0.4127
\end{array}\right] ; B_{22}=\left[\begin{array}{r}
0 \\
0 \\
0.1750
\end{array}\right] ; \\
& A_{31}=\left[\begin{array}{rrr}
0 & 1.0000 & 0 \\
0 & -0.6250 & -39.2699 \\
0 & 1.2580 & -0.4127
\end{array}\right] ; B_{31}=\left[\begin{array}{r}
0 \\
0 \\
0.0974
\end{array}\right] ; \\
& A_{32}=\left[\begin{array}{rrr}
0 & 1.0000 & 0 \\
0 & -0.6250 & -39.2699 \\
0 & 0.4296 & -0.4127
\end{array}\right] ; B_{32}=\left[\begin{array}{r}
0 \\
0 \\
0.1575
\end{array}\right] \text {. }
\end{aligned}
$$

The mode indicator membership functions $m_{i}(),. i=1,2,3$ are crisp functions which represent the operating modes, in this case $m_{i}(z)=1$, if $r=i$ and $m_{i}(z)=0$, otherwise. The normalized membership functions $n_{i j}(),. j=1,2$ describe the range of the state variables $x_{1}$ and $x_{3}$ in each mode as shown in Figure 3 and are obtained from standard membership functions available in the Fuzzy Logic Toolbox of Matlab. A suitable range for the state variables can be determined by constraining $x_{1}$ in the interval $[\pi / 5,3 \pi / 5]$.
Thus, the fuzzy modeling for the SMIB power system (38) is given by

Mode 1:

If $z$ is " 1.2144 p.u."

Then

Rule 1:

If $x_{1}$ is "about $\pi / 5 \mathrm{rad} / \mathrm{s}$ " and

$x_{3}$ is "closer to 1.089 p.u."

Then $\dot{x}=A_{11} x+B_{11}$

Rule 2:

If $x_{1}$ is "about $3 \pi / 5 \mathrm{rad} / \mathrm{s}$ " and

$x_{3}$ is "far from 1.089 p.u."

Then $\dot{x}=A_{12} x+B_{12}$

Mode 2:

If $z$ is " 1.1040 p.u."

Then

Rule 1:

If $x_{1}$ is "about $\pi / 5 \mathrm{rad} / \mathrm{s}$ " and

$x_{3}$ is "closer to 0.9 p.u."

Then $\dot{x}=A_{21} x+B_{21}$

Rule 2:

If $x_{1}$ is "about $3 \pi / 5 \mathrm{rad} / \mathrm{s}$ " and $x_{3}$ is "far from 0.9 p.u."

Then $\dot{x}=A_{22} x+B_{22}$

Mode 3:

If $z$ is " 0.9936 p.u."

Then

Rule 1:
If $x_{1}$ is "about $\pi / 5 \mathrm{rad} / \mathrm{s}$ " and $x_{3}$ is "closer to 0.729 p.u."
Then $\dot{x}=A_{31} x+B_{31}$

Rule 2:
Therefore, using $\left(A_{i j}, B_{i j}, \Pi\right), i=1,2,3, j=1,2$, we obtain the feedback gains for the stabilization of the SMIB power system (38) by solving the LMI's in Proposition 1 using the LMI Control Toolbox of Matlab. In order to use performance indices in the stabilizing control design, we adopt decay rates $\alpha_{1}=5, \alpha_{2}=0$ and $\alpha_{3}=5$ and control input constraints $\gamma_{i}=6$ for $i=1,2,3$. We take the initial conditions as $x_{0}=\left[\begin{array}{llll}2 \pi / 5 & 0-0.01 & 0.9\end{array}\right]$ and $r_{0}=1.1040$. Simulation results obtained with different stabilizing control designs given in Section 4 are divided in two cases: Case 1 - SMIB power system with stabilizing fuzzy control and Case 2 - 

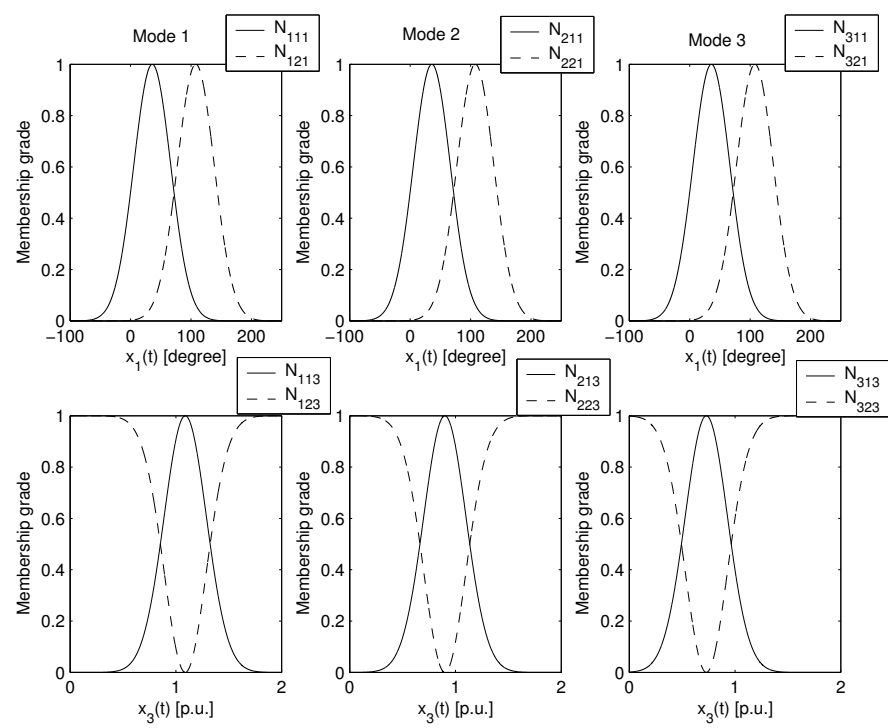

Figure 3: Membership functions adopted.

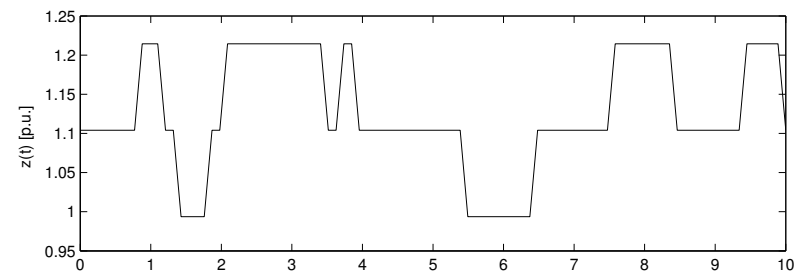

$t[s]$

Figure 4: Deviations in the infinite-bus voltage $z$ following the transition rate matrix $\Pi$.

SMIB power system with stabilizing fuzzy control + decay rate + input control constraint. In both cases, we use the software provided in Waner and Costenoble (2002) to simulate $z$ which is shown in Figure 4 for a period of time. Figures 5 and 6 show the main system responses and Table 1 presents the control design results for both Cases 1 and 2 .

The obtained results are comparable to the results presented in Guo et al. (2001). Note that, in both Cases 1 and 2 the system stabilization is satisfactory. In Case 2, the use of constraints in the stabilizing control design reduces the fluctuations in both state variables and control input. The advantage of using Markov jump systems to model the SMIB power system can be clearly seen as we include in the SMIB power system a more refined description of the infinite-bus voltage as compared to that used in Guo et al. (2001). For instance, there, one considers a constant value for the infinite-bus voltage and the changes in the external load during time are not represented. Taking into account the information on how the
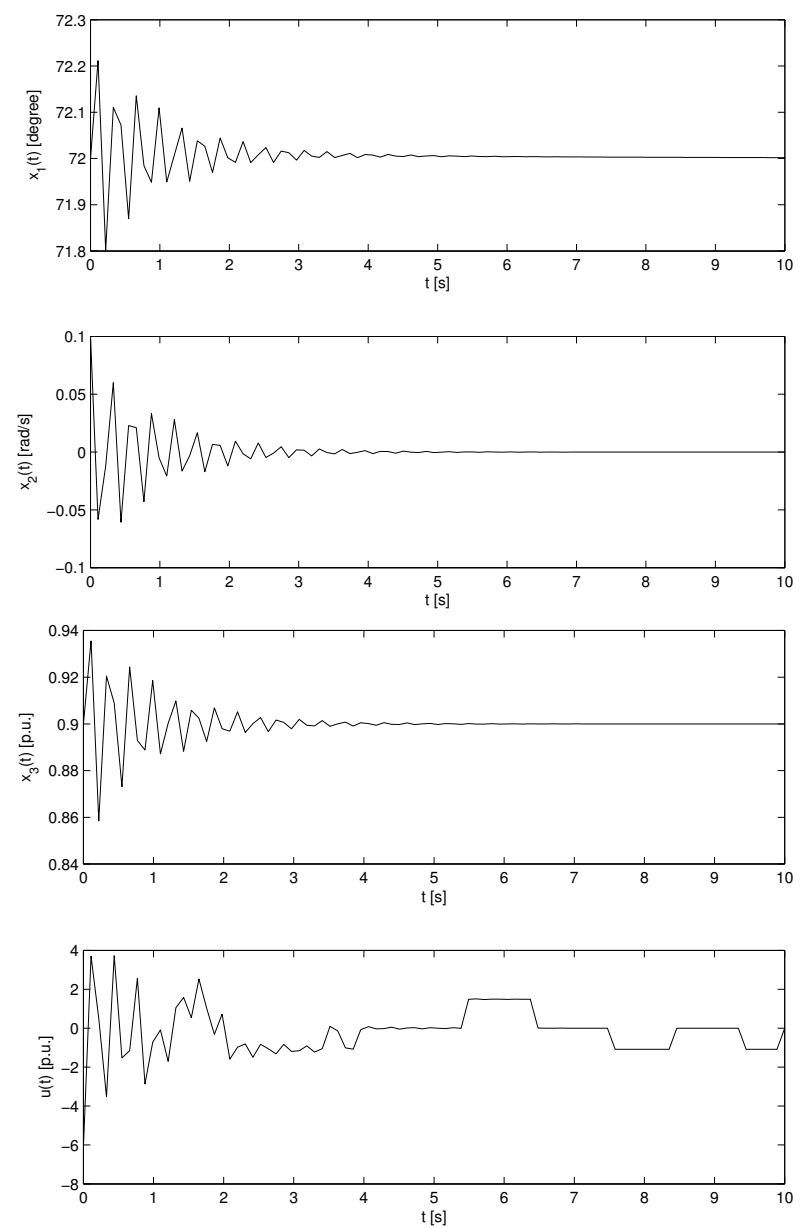

Figure 5: Case 1 - SMIB power system state variables and control.

infinite-bus voltage can vary, we can provide less restrictive conditions for the system stability using controllers with better performance. Another important point concerns the stability of the SMIB power system. Using the technique proposed in Guo et al. (2001), the system must be stable for all deviations in the infinite-bus voltage, whereas in the stochastic stability framework, stability of all operation modes is not even required.

\section{CONCLUDING REMARKS}

This paper presents a systematic fuzzy-model-based-control design for a class of nonlinear systems with Markovian jump parameters which employees recently developed fuzzy control techniques formulated in the context of LMI's. The class of systems considered is represented by a fuzzy system with two levels in its structure, one to represent the system modes and the other the nonlinearities in the system state. In this approach, the number of inference rules is directly related to 
Table 1: Control design results.

\begin{tabular}{|c|c|c|c|c|}
\hline Mode & \multicolumn{4}{|c|}{ Case 1} \\
\hline \multirow{2}{*}{1} & $F_{11}=$ & -13.9060 & -56.9870 & 5.2482 \\
\hline & $F_{12}=$ & -9.2534 & -43.2570 & 6.5994 \\
\hline \multirow{2}{*}{2} & $F_{21}=$ & -14.5150 & -60.7340 & 6.5752 \\
\hline & $F_{22}=$ & -9.6708 & -45.2000 & 8.0468 \\
\hline \multirow{2}{*}{3} & $F_{31}=$ & -15.3360 & -65.5510 & 8.2105 \\
\hline & $F_{32}=$ & -10.2320 & -47.8480 & 9.8305 \\
\hline Mode & \multicolumn{4}{|c|}{ Case 2} \\
\hline \multirow{2}{*}{1} & $F_{11}=$ & $-8.64 \times 10^{-}$ & $\begin{array}{ll}-9 & 0.8244\end{array}$ & 7.7447 \\
\hline & $F_{12}=$ & $2.02 \times 10^{-9}$ & -1.1686 & 7.1610 \\
\hline \multirow{2}{*}{2} & $F_{21}=$ & $-7.58 \times 10^{-}$ & $-9 \quad 0.7866$ & 7.5597 \\
\hline & $F_{22}=$ & $-5.58 \times 10^{-}$ & $\begin{array}{cc}9 & -1.0637\end{array}$ & 6.9946 \\
\hline \multirow[b]{2}{*}{3} & $F_{31}=$ & $-4.82 \times 10^{-}$ & $9 \quad 0.6616$ & $6.7462]$ \\
\hline & $F_{32}=$ & $-5.20 \times 10^{-}$ & $-9 \quad-0.8643$ & 6.2894 \\
\hline
\end{tabular}

the nonlinear system complexity and the number of LMI conditions is basically a combination of the number of inference rules of the fuzzy system and the number of inference rules of the fuzzy control. The number of inference rules can be reduced using local approximations of the nonlinear system but stability of the feedback nonlinear system is not guaranteed. In this paper we use local approximations to build the MJFS which represents the class of MJNLS considered. By heuristically choosing regions of the subspace that better represent the dynamics of the MJNLS we guarantee the convergence of the solutions reducing the approximation errors.

A fuzzy-model-based control law is used to stabilize the MJFS and then, the stochastic stability and stabilizability concepts are used to formulate the control design in the context of LMI's. The advantage of this approach can be clearly seen, for instance, we could consider in the fuzzy modeling a more refined description of the parameter variations in the nonlinear system. Taking into account this, we give less restrictive conditions for stability using a coupled Lyapunov function resulting in controllers which provide better performance.

Another important point concerns the stochastic stability. In comparison with the conventional techniques in the deterministic sense, stability of all system modes is not even required. In the proposed approach, when $u=0$, stability in each system mode is given in terms of the matrices $\left(A_{i j}, \Pi\right)$, $i \in \mathbb{S}, j=1,2, \ldots, R$, that is, stability in each mode is verified whenever $\operatorname{Re}\left\{\lambda\left[A_{i j}-\frac{1}{2} \pi_{i} I\right]<0, \pi_{i} \geq 0\right.$ whereas in the conventional techniques, stability in each mode is verified only if $\operatorname{Re}\left\{\lambda\left[A_{i j}\right]\right\}<0$.

Future work include the design of robust fuzzy controllers to consider in the control design the approximation error between the fuzzy-model-based system and the nonlinear sys-
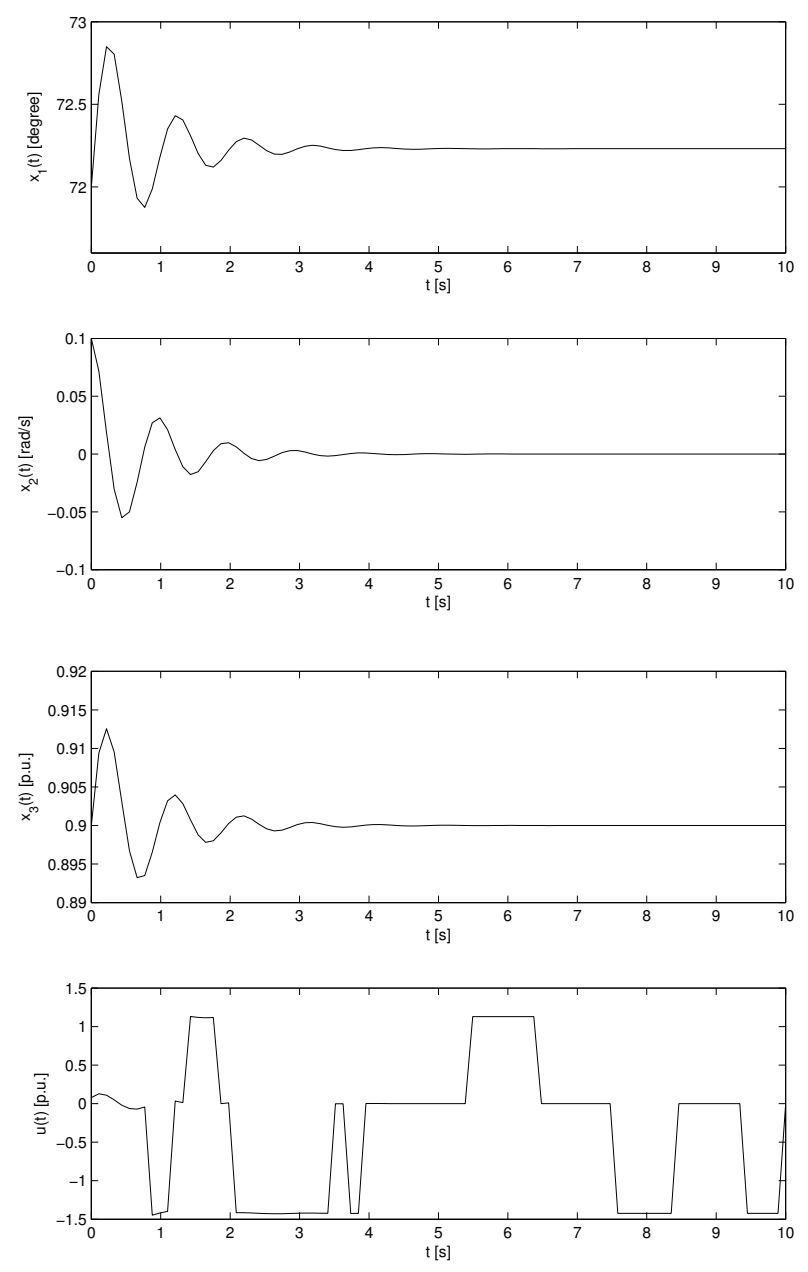

Figure 6: Case 2 - SMIB power system state variables and control.

tem and the development of a dynamic feedback controller to consider incomplete information of the system state.

\section{ACKNOWLEDGMENTS}

This work was supported by the Fundação do Amparo à Pesquisa do Estado de São Paulo (FAPESP) under grant 00/05060-1 and by the Conselho Nacional de Desenvolvimento Cientifico e Tecnológico under grant 473932/2003-2 Científico e . The authors would like to thank the reviewers for their valuable comments and sugestions

\section{REFERENCES}

Arrifano, N. S. D. and Oliveira, V. A. (2002a). Guaranteed cost fuzzy controllers for a class of uncertain nonlinear dynamic systems, Proc. XIV Congresso Brasileiro de Automatica, Natal, RN, pp. 1873-1877. 
Arrifano, N. S. D. and Oliveira, V. A. (2002b). Synthesis of an LMI-based fuzzy control system with guaranteed cost performance: a piecewise approach, Proc. XIV Congresso Brasileiro de Automatica, Natal, RN, pp. 2981-2986.

Boukas, E. K. and Liu, Z. K. (2001). Suboptimal design of regulators for jump linear system with time-multiplied quadratic cost, IEEE Transactions on Automatic Control 46(1): 944-949.

Boukas, E. K., Liu, Z. K. and Al-Sunni, F. (1999). Guaranteed cost control of markov jump uncertain system with time-multiplied cost function, Proc. 38th Conference Decision and Control, pp. 4125-4130.

Boukas, E. K. and Yang, H. (1999). Exponential stabilizability of stochastic systems with markovian jump parameters, Automatica 35(9): 1437-1441.

Boyd, S., Ghaoui, L. E., Feron, E. and Balakrishnan, V. (1994). Linear matrix inequalities in system and control theory, Philadelphia : SIAM.

Cao, S. G., Rees, N. W. and Feng, G. (1996). Analysis and design of a class of continuous time fuzzy control systems, International Journal of Control 64(6): 10691087.

Cao, S. G., Rees, N. W. and Feng, G. (1997). Analysis and design for a class of complex control systems, part ii: Fuzzy controller design, Automatica 33(6): 1029-1039.

Costa, O. L. V. and Boukas, E. K. (1998). Necessary and sufficient condition for robust stability and stabilizability of continuous-time linear systems with markovian jumps, Journal of Optimization Theory and Applications 99(2): 359-379.

Costa, O. L. V., do Val, J. B. R. and Geromel, J. C. (1999). Continuous-time state-feedback h2-control of markovian jump linear systems via convex analysis, Automatica 35(2): 259-268.

Farias, D. P., Geromel, J. C., do Val, J. B. R. and Costa, O. L. V. (2000). Output feedback control of markov jump linear systems in continuous-time, IEEE Transactions on Automatic Control 45(5): 944-949.

Guo, Y., Hill, D. J. and Wang., Y. (2001). Global transient stability and voltage regulation for power systems, IEEE Transactions on Power Systems 16(4): 678-688.

Ji, Y. and Chizeck, H. J. (1990). Controllability, stabilizability, and continuous-time markovian jump linear quadratic control, IEEE Transactions on Automatic Control 35(7): 777-788.
Khalil, H. (1996). Nonlinear systems, USA: Macmillan Publishing Company.

Krasovskii, N. N. and Lidskii, E. A. (1961). Analytical design of controllers in systems with random attributes I, II, III, Automation Remote Control 22: 1021-1025, 1141-1146, 1289-1294.

Kushner, H. J. (1967). Stochastic stability and control, New York: Academic.

Li, J., Wang, O. H., Niemann, D. and Tanaka, K. (2000). Dynamic parallel distributed compensation for TakagiSugeno:an LMI approach, Information Sciences 123(34): 201-221.

Mariton, M. (1990). Jump linear systems in automatic control, New York: Marcel Dekker.

Mariton, M. and Bertrand, P. (1985a). Output feedback for a class of linear systems with stochastic jumping parameters, IEEE Transactions on Automatic Control 30(9): 898-900.

Mariton, M. and Bertrand, P. (1985b). Robust jump linear quadratic control: a mode stabilizing solution, IEEE Transactions on Automatic Control 30(11): 1145-1147.

Nascimento, R. R., Oliveira, V. A., Arrifano, N. S. D., Gesualdo, E. and Tosetti, J. P. V. (2002). Control of the molten steel level in a strip-casting process using fuzzy T-S models, Proc. 15th Triennial World Congress of the International Federation of Control, Barcelona, Spain, number 02710 .

Rishel, R. (1975). Dynamic programming and minimum principles for systems with jump markov disturbances, SIAM Journal on Control 13(2): 338-371.

Sworder, D. D. (1969). Feedback control of a class of linear systems with jump parameters, IEEE Transactions on Automatic Control 14(1): 9-14.

Takagi, T. and Sugeno, M. (1985). Fuzzy identification of systems and its application to modeling and control, IEEE Transactions on Systems, Man and Cybernetic 15(1): 116-132.

Tanaka, K., Hori, T. and Wang, H. O. (2003). A multiple Lyapunov function approach to stabilization of fuzzy control systems, IEEE Transactions on Fuzzy Systems 11(4): 582-589.

Tanaka, K., Ikeda, T. and Wang, H. O. (1998). Fuzzy regulators and fuzzy observers: relaxed stability conditions and LMI-based designs, IEEE Transactions on Fuzzy Systems 8(2): 250-265. 
Tanaka, K., Iwasaki, M. and Wang, H. O. (2000). Stability and smoothness conditions for switching fuzzy systems, Proc. American Control Conference, pp. 24742478.

Tanaka, K. and Wang, H. O. (2001). Fuzzy control systems design and analysis: a linear matrix inequality approach, New York: John Wiley and Sons.

Teixeira, M. C. M., Assunção, E. and Avellar, R. G. (2003). On Relaxed LMI-Based Designs for Fuzzy Regulators and Fuzzy Observers, IEEE Transactions on Fuzzy Systems 11(5): 613-623.

Teixeira, M. C. M., Pietrobom, H. C. and Assunção, E. (2000). Novos resultados sobre a estabilidade e controle de sistemas não-lineares utilizando modelos fuzzy e LMI, Revista SBA - Controle \& Automação 11(1): $37-48$.

Teixeira, M. C. M. and Żak, S. H. (1999). Stabilizing controller design for uncertain nonlinear systems using fuzzy models, IEEE Transactions on Fuzzy Systems 15(1): 116-132.

Waner, S. and Costenoble, S. R. (2002). Markov system simulation, http://people.hofstra.edu/ faculty/ StefanWaner/ RealWorld/ markov/ markov.

Wang, H. O., Tanaka, K. and Griffin, M. F. (1996). An approach to fuzzy control of nonlinear: stability and design issues, IEEE Transactions on Fuzzy Systems 4(1): 14-23.

\section{A LOCAL LINEAR REPRESENTATIONS FOR THE SMIB POWER SYSTEM}

Consider the SMIB power system (38) which is repeated here for easy reference

$$
\dot{\xi}=f\left(\xi+x_{e}, r\right)+g\left(\xi+x_{e}, r\right) v
$$

where

$$
f\left(\xi+x_{e}, r\right)=\left[\begin{array}{lll}
f_{1} & f_{2} & f_{3}
\end{array}\right]^{T}
$$

and

$$
g\left(\xi+x_{e}, r\right)=\left[\begin{array}{lll}
0 & 0 & g_{3}
\end{array}\right]^{T}
$$

are vectorial functions with

$$
\begin{aligned}
& f_{1}=\xi_{2} \\
& f_{2}=-\frac{D}{2 H} \xi_{2}+\frac{\omega_{0}}{2 H}\left(\xi_{3}+0.9\right)
\end{aligned}
$$

$$
\begin{aligned}
f_{3}= & \frac{x_{d s}}{x_{d s}^{\prime} T_{d o}^{\prime}}\left[T_{d o}^{\prime}\left(x_{d}-x_{d}^{\prime}\right)\left(\frac{z \sin \left(\xi_{1}+2 \pi / 5\right)}{x_{d s}}\right)^{2} \xi_{2}\right] \\
& -\left(\frac{x_{d s}}{x_{d s}^{\prime} T_{d o}^{\prime}}-\frac{\cos \left(\xi_{1}+2 \pi / 5\right)}{\sin \left(\xi_{1}+2 \pi / 5\right)} \xi_{2}\right)\left(\xi_{3}+0.9\right) \\
g_{3}= & \frac{x_{d s}}{x_{d s}^{\prime} T_{d o}^{\prime}}\left(\frac{z \sin \left(\xi_{1}+2 \pi / 5\right)}{x_{d s}}\right) k_{c}
\end{aligned}
$$

$\xi=x-x_{e}$ and $v=u-u_{e}$ the new system coordinates, with $x_{e}=\left[\begin{array}{lll}2 \pi / 5 & 0 & 0.9\end{array}\right]^{T}$ and $u_{e}=0$.

Let mode at time $t$ be $i$, i.e., $r=i, i \in \mathbb{S}$ and $\bar{x}$ be a linearization point not necessarily an equilibrium point. Following Teixeira and Żak (1999), the objective is to obtain matrices $A_{i}$ and $B_{i}$ such that in the vicinity of $\bar{x}$ we have

$$
f\left(\xi+x_{e}, i\right)+g(\xi+x e, i) v \approx A_{i} \xi+B_{i} v
$$

and

$$
f\left(\bar{x}+x_{e}, i\right)+g(\bar{x}+x e, i) v \approx A_{i} \bar{x}+B_{i} v .
$$

Since $v$ is arbitrary, we have $g(\bar{x}+x e, i)=B_{i}$. The columns of the matrix $A_{i}$ are given by the formula

$$
a_{k}=\nabla f_{k}(\bar{x})+\frac{f_{k}(\bar{x})-\bar{x}^{T} \nabla f_{k}(\bar{x})}{\|\bar{x}\|^{2}} \bar{x}
$$

for $\bar{x} \neq 0$ and $k=1,2,3$ where $\nabla f_{k}(\bar{x}): \mathbb{R}^{n} \rightarrow \mathbb{R}^{n}$ is the gradient, a column vector, of $f_{k}$ evaluated at $\xi$. We can use function jacobian available in the Symbolic Math Toolbox of Matlab in order to compute the gradient for the SMIB power system.

The Teixeira \& Żak linearization formula produces linear representations instead of affine, usually obtained using the Taylor linearization formula. In order to verify this statement, consider the Taylor linearization formula

$$
A_{i}=\nabla f(\bar{x}):=\left.\frac{\partial f(\xi+x e, i)}{\partial \xi}\right|_{\xi=\bar{x}} .
$$

The representation of a function $f(\cdot, \cdot)$ around $\bar{x}$ is thus given by

$$
f\left(\xi+x_{e}, i\right) \approx f\left(\bar{x}+x_{e}, i\right)+A_{i}(\xi-\bar{x}) .
$$

Thus, whenever $f(\bar{x}+x e, i) \neq 0$ which occurs if $\bar{x}$ is not an equilibrium point, this representation produces affine models instead of linear models, as mentioned. Hence, using the Teixeira \& Żak linearization formula, we can obtain several local linear approximations $\left(A_{i j}, B_{i j}\right), i=1, \ldots, N, j=$ $1,2, \ldots, R$ of a nonlinear system in any chosen linearization points and then build a fuzzy system representation. 